\title{
CARACTERIZAÇÃO DOS RESÍDUOS SÓLIDOS NA UFT, CAMPUS GURUPI- TO
}

\author{
Lamia Mahmud Fawzi ${ }^{1}$, Leila Francisca Dias ${ }^{1}$, Jaci de Sousa Dias ${ }^{1,2}$, Virgílio \\ Lourenço da Silva Neto ${ }^{3}$, Priscila Bezerra de Souza ${ }^{4}$
}

1. Discente do Curso de Ciências Biológicas da Universidade Federal do Tocantins, Campus Gurupi.

2. Técnica em Laboratório da Universidade Federal do Tocantins-UFT

3. Msc em Ciências Florestais e Ambientais, Professor Instituto Federal de

Educação, Ciências e Tecnologia, Campus Dianópolis. E-mail:

virgilio.neto@ifto.edu.br

4. Professora adjunta III da Universidade Federal do Tocantins, Coordenadora do

Curso de Graduação em Ciências Biológicas da UFT, Campus Gurupi

Recebido em: 03/10/2016 - Aprovado em: 21/11/2016 - Publicado em: 05/12/2016

DOI: 10.18677/EnciBio 2016B 108

\begin{abstract}
Resíduos sólidos resultam de processos de diversas atividades cotidianas e são considerados, muitas vezes, sem utilidade para as pessoas ou para o sistema de produção. Objetivou-se caracterizar e quantificar os resíduos sólidos gerados na UFT, campus de Gurupi-TO. Para a caracterização e quantificação dos resíduos gerados nos setores Casadinho, Biblioteca, Restaurante, Cantina, Laboratório multiuso, Banheiros, Administração e Parfor no período de 23 a 30 de junho de 2016, quando foram realizadas coletas dos resíduos e posteriormente pesagem dos mesmos foram calculadas médias diárias, estimativa da média mensal e a composição gravimétrica. Foi estimado que a quantidade total de resíduos gerados semanalmente foi de $763,45 \mathrm{Kg}$, o equivalente a $127,2 \mathrm{~kg} /$ dia e uma produção per capta de $0,527 \mathrm{~kg}$. Considerando apenas os dias úteis, a quantidade de resíduos produzidos foi de $727,83 \mathrm{Kg}$ equivalentes a aproximadamente $145,5 \mathrm{~kg} / \mathrm{dia}$, o que corresponde a $0,100 \mathrm{~kg}$.habitante ${ }^{-1} \mathrm{dia}^{-1}$. Quanto ao sábado, a quantidade de resíduo produzido foi de $35,63 \mathrm{~kg}$, sendo produzido um valor de $0,024 \mathrm{~kg}$.habitante ${ }^{-1} \mathrm{dia}^{-1}$.
\end{abstract}

RESUMO

PALAVRAS-CHAVE: educação ambiental, reciclagem, sustentabilidade.

\section{THE SOLID WASTE CHARACTERIZATION AT THE FEDERAL UNIVERSITY OF TOCANTINS, CAMPUS GURUPI}

\begin{abstract}
The solid waste result from processes of various daily activities and are considered often useless to the people or to the production system. The aim of this study was to characterize the solid waste generated campus Gurupi-TO the Federal University of Tocantins. For the characterization and quantification of waste generated in the following sectors: Casadinho, Library, Restaurant, Canteen, Laboratory, Bath, Administration and Parfor from 23 to 30 June 2016, when they were made of waste collection and then weighing the same were calculated daily average, estimated average monthly and gravimetric composition. It has been estimated that the total
\end{abstract}


amount of waste generated was $763,45 \mathrm{Kg}$ weekly, equivalent to $127.2 \mathrm{~kg} /$ day and the production of $0.527 \mathrm{~kg}$ per capita. Considering only the working days, the amount of waste produced was $727,83 \mathrm{Kg}$ equivalent to approximately $145.5 \mathrm{~kg} \mathrm{/} \mathrm{day,}$ corresponding to $0.100 \mathrm{~kg} \cdot$ habitante $^{-1} \mathrm{dia}^{-1}$. As to Saturday, the amount of waste produced was $35,63 \mathrm{~kg}$ being produced a value of $0.024 \mathrm{~kg}$. habitante ${ }^{-1} \mathrm{dia}^{-1}$.

KEYWORDS: environmental education, recycling, sustainability.

\section{INTRODUÇÃO}

Resíduos sólidos resultam de processos de diversas atividades cotidianas e são considerados, muitas vezes, sem utilidade para as pessoas ou para o sistema de produção. Entretanto, podem ser convertidos em matéria prima para a produção de novos produtos substituindo os recursos naturais como fonte e a energia contida pode ser reaproveitada, o que coloca em destaque programas relativos ao gerenciamento de resíduos, fundamentados em normas e leis relacionadas à coleta, acondicionamento e destinação final (MESQUITA et al., 2011).

A população humana para realização de suas atividades, gera uma série de resíduos, porém, até antes da II Guerra Mundial estes resíduos eram de composição mais simples, basicamente orgânica e de fácil destinação e decomposição. A geração per capita de resíduos está diretamente relacionada ao consumo exagerado, rápido crescimento populacional e adensamento espacial. A evolução dos materiais empregados pelas sociedades, acompanhando o modelo de industrialização iniciado na Revolução Industrial (FIGUEIREDO, 1995), vem tornando cada vez mais complexo o tratamento e destinação final destes resíduos (JULIATTO et al., 2011).

Portanto, uma das alternativas de alertar a sociedade é através da Educação Ambiental onde busca-se abrir os olhos e a mente das pessoas que ainda não conseguiram entender a gravidade da situação em que o meio ambiente se encontra, mostrando que o homem é apenas mais um membro da natureza em que vive e não superior a esta, contrapondo as ideias que o colocaram como centro do universo e esquecendo-se da importância dos demais componentes da natureza (MARINHO et al., 2014). A Política Municipal de Resíduos Sólidos deve ser elaborada visando à conservação in situ: conservação de ecossistemas e habitats naturais, manutenção e recuperação de populações viáveis de espécies em seus meios naturais (GURUPI, 2013).

A NBR 10.004/04 define resíduos sólidos como: Resíduos nos estados sólido e semissólido resultantes das atividades de origem industrial, doméstica, hospitalar, comercial, agrícola e serviços de varrição. Estão inclusos nesta definição os lodos provenientes de sistemas de tratamento de água, aqueles gerados em equipamentos e instalações de controle de poluição, bem como determinados líquidos cujas particularidades tornem inviável o lançamento na rede pública de esgotos ou corpos de água, ou exijam para isso soluções técnicas e economicamente inviáveis em face à melhor tecnologia disponível (ABNT 2004).

A elevação do grau de conhecimento está no âmago do processo de desenvolvimento das Instituições de Ensino Superior-IES. A educação superior é, portanto, uma Responsabilidade Social de primeira ordem e pode ser fortalecida significativamente entre o Estado, as Instituições de Ensino Superior e a sociedade, provido de uma reflexão sobre os problemas sociais que refletem a sociedade (LOHN, 2011). Para transformar o pensamento e formar cidadãos capazes de enfrentar os problemas atuais, faz-se necessário a reforma do ensino nas Universidades (GARBIN et al., 2015). 
Alguns estudos similares foram realizados no âmbito da caracterização de resíduos sólidos no campus Bela Vista do Instituto Federal de Mato Grosso por ALVES et al., (2013); MENEZES (2014), que avaliaram a gestão de resíduos sólidos no programa da USP Recicla, no campus São Carlos, JULIATTO et al. (2011), abordaram o papel fundamental das Universidades como laboratório de ideias na constante busca por soluções para o desenvolvimento sustentável.

De acordo com o Decreto № 5.940 , de 25 de outubro de 2006, a separação dos resíduos recicláveis descartados pelos órgãos e entidades da administração pública federal direta e indireta na fonte geradora e a destinação às associações e cooperativas dos catadores de materiais recicláveis são reguladas pelas disposições deste Decreto, que considera resíduos recicláveis descartados os materiais passíveis de retorno ao ciclo produtivo, rejeitados pelos órgãos e entidades da administração pública federal direita e indireta (BRASIL, 2006).

Estudos sobre a caracterização de resíduos na UFT, campus de Gurupi ainda são escassos ou incipientes promovendo assim uma lacuna de informações técnicas e científicas. Portanto, objetivou-se caracterizar e quantificar os resíduos sólidos gerados na UFT, campus de Gurupi-TO.

\section{MATERIAL E MÉTODOS}

A pesquisa foi realizada na Universidade Federal do Tocantins, campus de Gurupi- TO a 280 m de altitude sob as coordenadas $142945.0564^{\prime}$ latitude e $51^{\circ}$ 19' 41.25W (Figura 1).

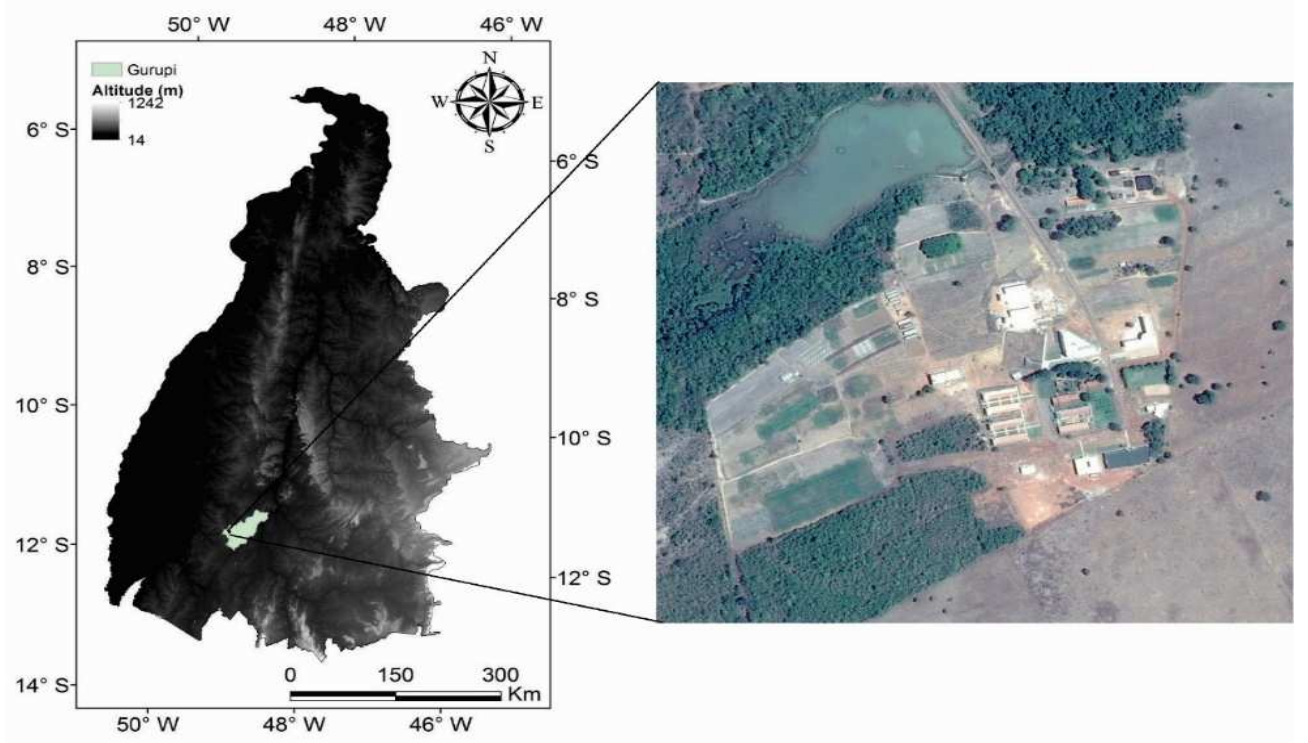

(Fonte: Autores, 2016)

(Google, MapLink Imagens, 2013)

FIGURA 1 - Modelo digital de elevação ASTER para o Estado do Tocantins com Datum Horizontal SAD 69 e localização do campus de Gurupi, Universidade Federal do Tocantins.

O campus de Gurupi está inserido no bioma Cerrado, fitofisionomia cerrado sensu stricto, em seu entorno estão presentes os córregos Mutuca e Água Franca, que abrigam parte da flora e fauna de Gurupi-TO. O campus de Gurupi constitui-se em um dos sete campi da Universidade Federal do Tocantins localizado na região sul do Estado, neste, oferecidos quatro cursos de graduação presencial: Agronomia, Engenharia Florestal, Química Ambiental e Engenharia de Bioprocessos e três cursos na modalidade EAD: Biologia, Física e Química modalidade licenciaturas. $\mathrm{Na}$ 
pós-graduação stricto sensu são oferecidos os cursos de Mestrado em Produção Vegetal, Mestrado em Biotecnologia, Mestrado em Ciências Ambientais e Florestais e Doutorado em Produção Vegetal.

Para atender aproximadamente 1.269 alunos, a UFT campus de Gurupi tem no quadro de funcionários 86 professores efetivos, sendo 63 doutores e 23 mestres, 68 servidores técnico-administrativos e ainda 24 colaboradores temporários que prestam serviços terceirizados de limpeza e jardinagem do campus.

Foi realizada a coleta dos resíduos sólidos no dia 22/08/2015 apenas em um dia de sábado e entre os dias 23 a 30 de junho de 2016, em dias úteis, sendo que os mesmos eram coletados três vezes ao dia, posteriormente os resíduos sólidos gerados neste período foram pesados durante os sete dias úteis consecutivos, sendo calculada a média diária, a estimativa média mensal e a composição gravimétrica (MONTEIRO et al., 2011). A coleta dos resíduos foi realizada com auxílio dos funcionários terceirizados do campus de Gurupi, sendo os locais de coleta para quantificação os setores: Casadinho, Biblioteca, Restaurante, Cantina, Laboratório, Banheiros, Administração e Parfor.

A classificação dos resíduos sólidos foi feita de acordo com a ABNT, podendo ser classificados nas seguintes classes:

I - perigosos;

IIA - não perigosos; e

IIB - inertes.

Para a separação dos resíduos por turno foram usados alguns equipamentos de EPI como: luvas e bota, lona plástica preta, balança digital marca Camry EB9014 com capacidade até $25 \mathrm{~kg}$. Para a caracterização dos resíduos coletados por gravimetria foram separados e classificados em cinco grupos: Plásticos (rígidos e maleáveis), papéis (papel e papelão), metais, compostos orgânicos e outros (pano, vidro, pedra, ou seja, todos os outros resíduos não incluídos anteriormente com exceção dos resíduos líquidos do laboratório). A porcentagem relativa a cada grupo de resíduo foi calculada conforme GONÇALVES et al., (2010) :

$$
\text { Material (\%) }=\frac{\text { Peso da fraç̃ăo do material }(\mathrm{Kg}) \times 100}{\text { Peso total da amostra }(\mathrm{kg})}
$$

Após todo o processo de pesagem os resíduos foram depositados em um contêiner para serem coletados pelo serviço público de coleta, sendo que os resíduos do campus de Gurupi são coletados pelo serviço público de coleta (Prefeitura Municipal) duas vezes por semana, uma vez que no campus de Gurupi não há coleta seletiva. A produção de resíduos sólidos per capta foi estimada pela Equação 2:

$$
\text { Prs }=\frac{\text { T.rs }}{\text { T.pes }}
$$

Onde,

Prs é a produção de resíduos sólidos per capta $(\mathrm{kg})$

T.rs é o total de resíduos sólidos

T.pes é o total de pessoas que frequenta o campus. 
O mesmo procedimento foi realizado separadamente para a análise da geração de resíduos sólidos nos sábados letivos do campus, uma vez que a dinâmica deste dia é peculiar, podendo gerar inconsistência nas observações dos dados, para isso foram realizadas coletas em apenas um sábado letivo, para a análise dos resíduos sólidos nesse sábado foram identificados apenas quatro locais de geração de resíduo.

\section{RESULTADOS E DISCUSSÃO}

Foram coletados $763,45 \mathrm{Kg}$, o equivalente a $127,2 \mathrm{Kg} /$ dia e uma produção per capta de $0,527 \mathrm{~kg}$. Dentro da classificação dos resíduos a maior porcentagem encontrada foi para o resíduo orgânico, seguido pelo papel/papelão e plástico, já os resíduos do tipo vidro, metal e outros corresponderam a menos de $3 \%$ do total. $\mathrm{A}$ Figura 2 mostra a caracterização dos resíduos produzidos durante o período avaliado, constatou-se que o principal resíduo é de natureza orgânica $(74 \%)$, seguido por papel/papelão (18\%), metal, isopor e outros apresentaram baixos percentuais.

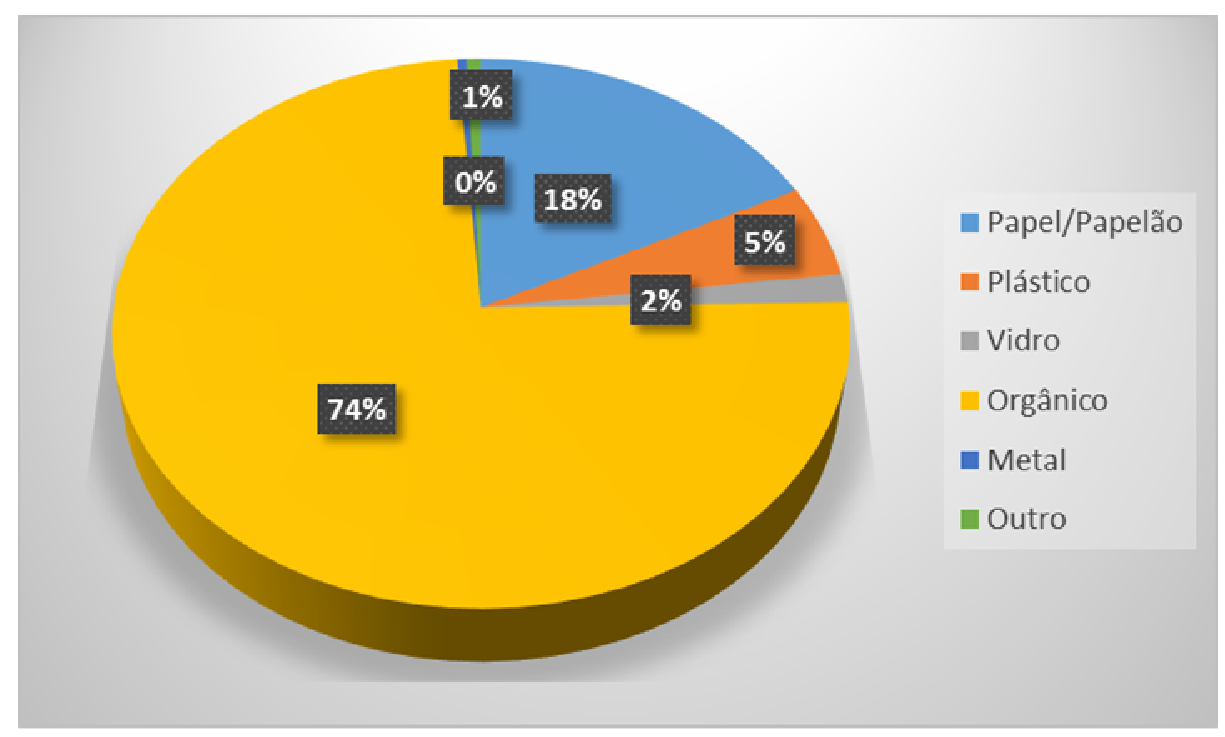

FIGURA 2 - Resíduos sólidos gerados na UFT, campus Gurupi-TO

Fonte: Autores

A cantina não é administrada pelo campus, mas é encarregada pela coleta dos resíduos gerados decorrentes das atividades, entretanto quem recolhe todos os resíduos é a prefeitura municipal de Gurupi-TO. Considerando apenas os dias úteis, a quantidade total de resíduos produzidos foi de $727,83 \mathrm{Kg}$ equivalentes a aproximadamente $145,5 \mathrm{~kg} / \mathrm{dia}$, o que corresponde a $0,100 \mathrm{~kg}$. habitante ${ }^{-1} \mathrm{dia}^{-1}$.

Quanto ao sábado, a quantidade de resíduo produzido foi de $35,63 \mathrm{~kg}$, comprovando a hipótese que este é o dia de menor dinâmica de funcionamento semanal do campus, sendo produzido um valor per capta de $0,024 \mathrm{~kg}$.habitante ${ }^{-1} \mathrm{dia}^{-}$ 1. Outra peculiaridade na observação realizada no sábado é o fato da cantina estar inativa, não sendo, portanto, gerado resíduo neste dia da observação. Assim, foram coletados resíduos sólidos somente nos setores: Casadinho, Anfiteatro, Incubadora e Laboratório próximo à Guarita (Figura 3). 


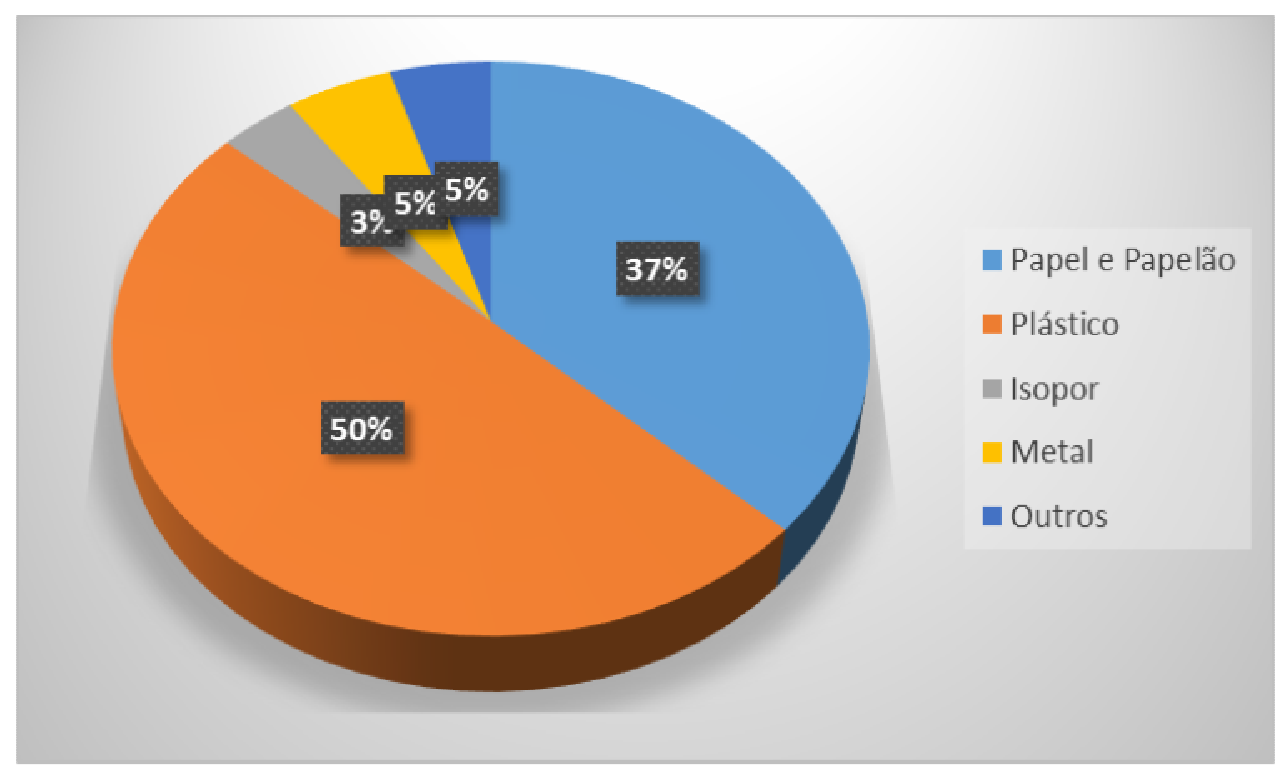

FIGURA 3 - Caracterização dos resíduos sólidos produzidos no

Fonte: Autores sábado letivo do campus Gurupi-TO.

No Brasil, em 2009, a massa coletada de resíduos sólidos domiciliares e públicos variou de $0,77 \mathrm{~kg}$.habitante ${ }^{-1} \mathrm{dia}^{-1}$ a $1,19 \mathrm{~kg}$.habitante ${ }^{-1} \mathrm{dia}^{-1}$ e a média foi de 0,96 kg.habitante ${ }^{-1} \mathrm{dia}^{-1}$ (CAMPOS, 2012). Com base nesses dados, é possível perceber que a média de geração de resíduos no campus de Gurupi, considerando todo o período de análise equivalente a $0,088 \mathrm{~kg} \cdot$ habitante ${ }^{-1} \mathrm{dia}^{-1}$, está abaixo da média nacional. No entanto, o resultado está acima do que foi observado por ALVES et al. (2013), em estudos sobre resíduos sólidos produzidos no campus Bela Vista do Instituto Federal de Mato Grosso, que encontrou média diária de 0,014 kg.habitante ${ }^{-1} \mathrm{dia}^{-1}$.

A Figura 4 demonstra a geração de resíduos sólidos coletadas por setor no campus Gurupi/UFT considerando apenas o sábado letivo. Deve-se levar em consideração que os ambientes geradores de matéria orgânica foram desconsiderados em função do não funcionamento da cantina e restaurante universitário neste dia. Observou-se que o Casadinho foi o setor com maior geração de resíduos sólidos, seguido pelos laboratórios próximos à guarita, anfiteatro e incubadora, respectivamente.

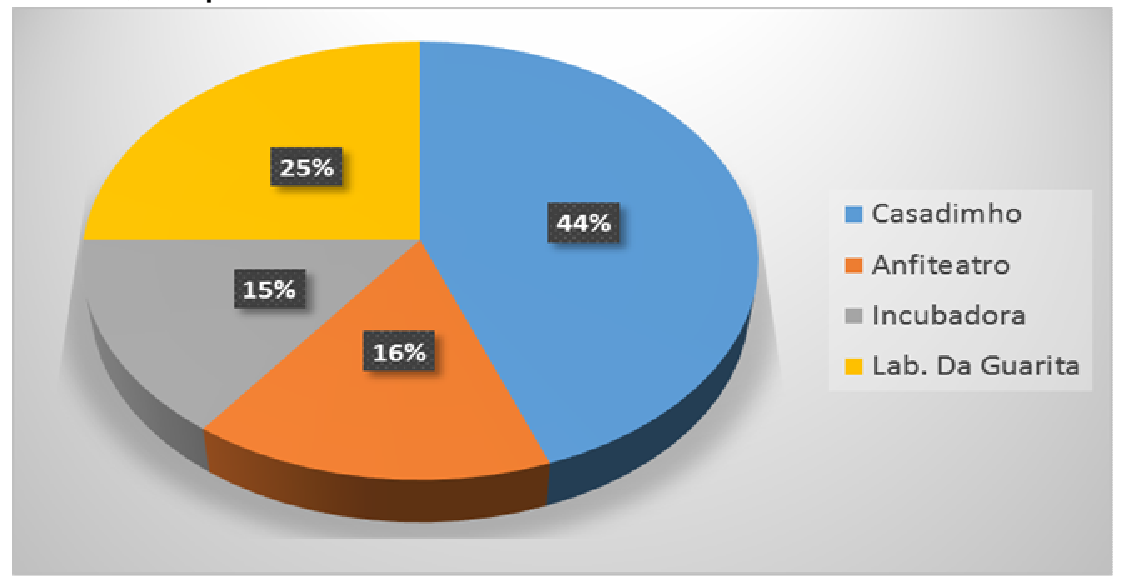

FIGURA 4 - Geração de Resíduos Sólidos por setor na coleta realizada ao sábado, dia Fonte: Autores 22/08/2015, dia de menor dinâmica do funcionamento do campus de Gurupi-TO. 
MESQUITA et al. (2011), estudaram a geração de resíduos sólidos no campus da Universidade Fumec, observaram que a produção total de resíduo por dia é de $342,75 \mathrm{~kg}$, e o número de alunos, professores e funcionários existentes por turno na Instituição, apresentaram média de 5.215 pessoas, sendo encontrado um valor da geração per capita de $0,0657 \mathrm{~kg}$.habitante ${ }^{-1}$ turno $^{-1}$, perfazendo uma média de $0,197 \mathrm{~kg}$.habitante ${ }^{-1} \mathrm{dia}^{-1}$. Tais resultados contrastam com a realidade do campus Gurupi, uma vez que a média de resíduos sólidos gerados considerando todo o período de análise foi equivalente a 127,2 kg/dia e 0,088 kg.habitante ${ }^{-1} \mathrm{dia}^{-1}$. A Figura 5 demonstra a distribuição dos resíduos sólidos produzidos no Casadinho do campus de Gurupi/UFT de maior geração de resíduos durante o sábado.

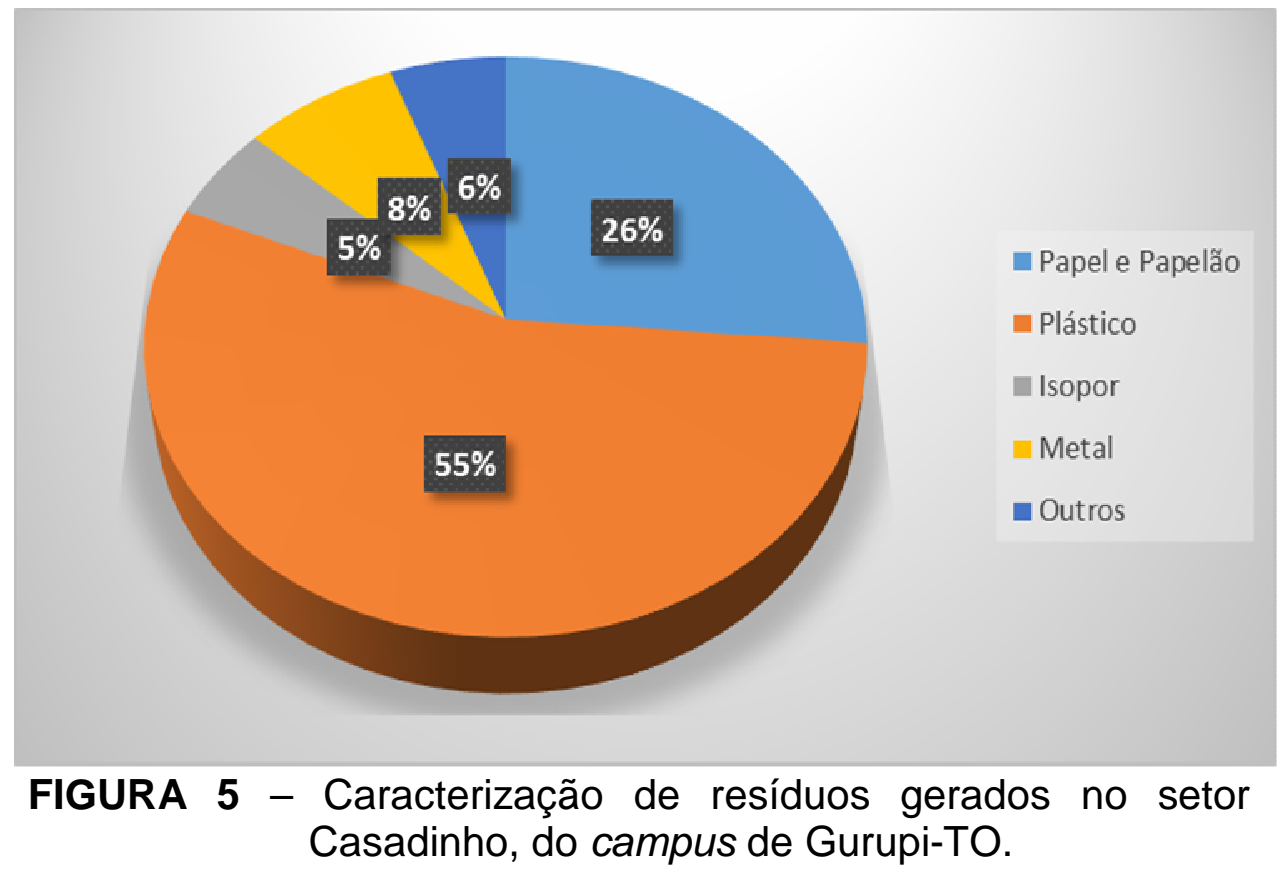

Fonte: Autores

A Figura 6 representa os tipos de resíduos sólidos produzidos no setor Anfiteatro onde se constatou a menor geração de resíduos. Neste setor, os resíduos gerados são basicamente plástico e papel/papelão, com $88 \%$ e $12 \%$, respectivamente. 


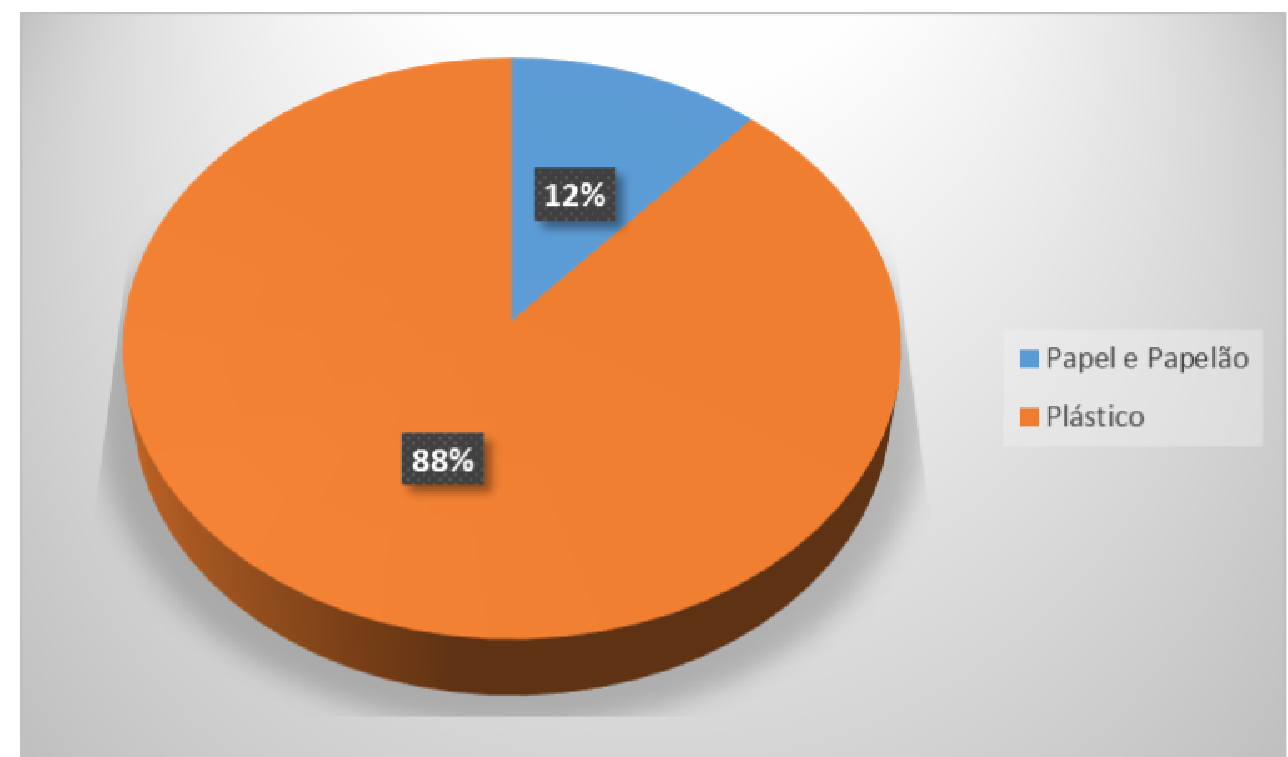

FIGURA 6 - Local de menor produção de resíduos sólidos setor

Fonte: Autores Anfiteatro, campus de Gurupi.

A Tabela 1 demonstra os tipos de resíduos sólidos coletados no dia 22/08/2015 e entre os dias 23 a 30 de junho de 2016 e sua respectiva classificação de acordo com a resolução 313/2002 do CONAMA e de acordo com as classes estabelecidas pela NBR 10.004/04 (CONAMA, 2002)

TABELA 1 - Resíduos sólidos coletados no campus de Gurupi da UFT e a respectiva classificação conforme CONAMA 313/2002 e NBR $10.004 / 04$

\begin{tabular}{|c|c|c|c|}
\hline Item & Resíduo & $\begin{array}{c}\text { Classe } \\
\text { (CONNAMA) }\end{array}$ & $\begin{array}{c}\text { Classe } \\
\text { (NBR 10.004) }\end{array}$ \\
\hline 1 & Papel/papelão & A006 & II-B \\
\hline 2 & Pequenas embalagens de plástico & A207 & II-B \\
\hline 3 & $\begin{array}{l}\text { Embalagens metálicas (latas } \\
\text { vazias) }\end{array}$ & A104 & II-B \\
\hline 4 & $\begin{array}{l}\text { Resíduos de madeira contendo } \\
\text { substâncias não tóxicas }\end{array}$ & A009 & II-B \\
\hline 5 & Resíduo do restaurante (orgânico) & A001 & II-A \\
\hline 6 & Resíduos de vidro & A117 & II-B \\
\hline 7 & $\begin{array}{l}\text { Outros resíduos não perigosos } \\
\text { (rejeitos) }\end{array}$ & A099 & II-A \\
\hline 8 & $\begin{array}{l}\text { Resíduos de laboratório - } \\
\text { Inorgânicos }\end{array}$ & D099 & I \\
\hline
\end{tabular}

I - Perigosos; II-A - Não perigosos e não-inertes; II-B - Não perigosos e inertes (Fonte: GONÇALVES, 2010. Adaptado)

É importante destacar que este estudo apresenta limitações com relação à caracterização do resíduo sólido na UFT, campus de Gurupi, principalmente em decorrência das dificuldades na aquisição e utilização de equipamentos necessários 
à pesagem dos resíduos, sendo recomendado estudos mais aprofundados para futuras análises dos resíduos produzidos neste campus universitário.

\section{CONCLUSÕES}

A quantidade gerada de resíduos sólidos revela que grande parte que é recolhida pela prefeitura é passível de reutilização, reciclagem ou compostagem, dessa forma pode-se inferir que o campus de Gurupi necessita urgentemente de um plano de gerenciamento de resíduos sólidos.

A coleta no campus Gurupi, apesar de possuir recipientes específicos para cada tipo de resíduo, não obedece a seletividade do resíduo sólido produzido no campus.

\section{REFERÊNCIAS}

ABNT - Associação Brasileira De Normas Técnicas - NBR 10004 - Resíduos sólidos - classificação. Rio de Janeiro: ABNT, 2004.

AlVES, T. O.; VAlENTINI, C. M. A.; PElEGRINE, R. A.; DE FARIA, G. Caracterização dos resíduos sólidos do IFMT-Campus Bela Vista. Enciclopédia Biosfera, v. 9, n. 17, p. 3292-3304, 2013. Disponível em: < http://www.conhecer.org.br/enciclop/2013b/MULTIDISCIPLINAR/caracterizacao\%20 do.pdf >

BRASIL. Decreto Presidencial no 5.940. Brasília: Casa Civil, 25 de outubro de 2006. 2 p.

CAMPOS, H. K. T. Renda e evolução da geração per capita de resíduos sólidos no Brasil. Engenharia Sanitária e Ambiental, v. 17, n. 2, p. 171-180, 2012. Disponível em: < http://www.scielo.br/pdf/esa/v17n2/a06v17n2 >

CONAMA. Resolução no 313/2002. Inventário Nacional de Resíduos Sólidos e Industriais: Conselho Nacional de Meio Ambiente Brasília, 2002.

FIGUEIREDO, P. J. M. A sociedade dolixo. Piracicaba: Editora Unimep, 1995. 240 p.

GARBIN, A.; GOMES, A.; SOUZA, M.; ARCIERI, R. M.; ROVIDA, T. A. S. et al. A responsabilidade socioambiental na formação acadêmica. $O$ Mundo da Saúde, São Paulo, v. 39, n. 1, p. 119-125, 2015. Disponível em: <>

GONÇALVES, M. S.; KUMMER, L.; SEJAS, M. I.; RAUEN, T. G.; BRAVO, C. E. C. Gerenciamento de resíduos sólidos na Universidade Tecnológica Federal do Paraná Campus Francisco Beltrão. Revista Brasileira de Ciências Ambientais-Número, n. 15 , p. 80, 2010. Disponível em: < http://abesdn.org.br/publicacoes/rbciamb/PDFs/15-09_RBCIAMB-N15-Mar-2010-

Materia07_artigos230.pdf >

GURUPI. Plano Municipal de Resíduos Sólidos 2013. Disponível em: < http://www.gurupi.to.gov.br/resources/arquivos-publicados/planos-

municipais/residuos_solidos_2013.pdf > 
JULIATTO, D. L.; CALVO, M. J.; CARDOSO, T. E. Gestão integrada de resíduos sólidos para instituições públicas de ensino superior. Revista Gestão Universitária na América Latina-GUAL, v. 4, n. 3, p. 170-193, 2011. ISSN 1983-4535. Disponível em: < http://stat.ijie.incubadora.ufsc.br/index.php/gual/article/viewFile/1262/1639 >

LOHN, V. M. Indicadores de responsabilidade social: uma proposta para as Instituições de Ensino Superior. Revista Gestão Universitária na América LatinaGUAL, v. 4, n. 1, p. 110-128, 2011. ISSN 1983-4535. Disponível em: <>

MARINHO, A. A.; MARQUES, M. L. A. P.; DA SILVA, A. F.; ARAÚJO, J. E. Q.; DA SILVA QUEIROZ, T. H. et al. A educação ambiental na formação da consciência ecológica. Caderno de Graduação-Ciências Exatas e Tecnológicas-UnitAlagoas, v. 1, n. 1, p. 11-18, 2014. ISSN 2357-9919. Disponível em: <>

MENEZES, C. M. V. M. D. Gestão de resíduos sólidos em Instituições de Ensino Superior: o programa USP Recicla no campus de São Carlos. 2014. PósGraduação (Mestrado). Programa de Mestrado Profissional em Administração e Gestão Ambiental e Sustentabilidade, Universidade Nove de Julho - UNINOVE 96 p.

MESQUITA, E. G.; FIUZA, M. S. S.; SARTORI, H. J. F. Gerenciamento de resíduos sólidos: Estudo de Caso em Campus Universitário. Construindo, v. 3, n. 1, p. 37-45, 2011. ISSN 2318-6127. Disponível em: < file://C:/Users/vyrgi/Documents/TCC\%20LAMIA\%20JACI\%20LEILA/Mesquita.pdf >

MONTEIRO, J. H. P. Manual de gerenciamento integrado de resíduos sólidos. Rio de Janeiro: IBAM, 2011. 200 p. 